\title{
Risk factors for hypertension among the adult Moroccan population
}

M.A. Tazi, ${ }^{1}$ S. Abir-Khalil, ${ }^{2}$ F. Lahmouz, ${ }^{1}$ M.L. Arrach ${ }^{1}$ and N. Chaouki ${ }^{1}$

$$
\begin{aligned}
& \text { عوامل اختطار فرط ضغط الدم بين البالغين في المملكة المغربية }
\end{aligned}
$$

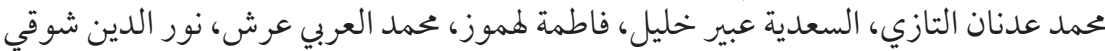

الخلاصسة: استمد الباحثون في هذه الدراسة المعطيات من المسح الوطني المغربي لعام 2000 للتعرٌّف على عو امل

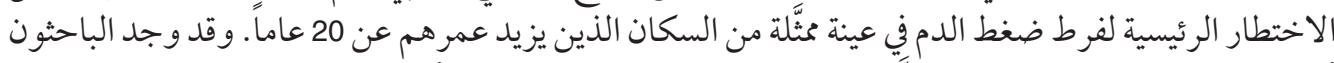

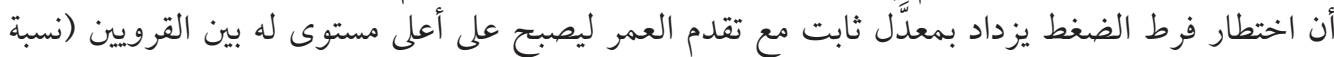

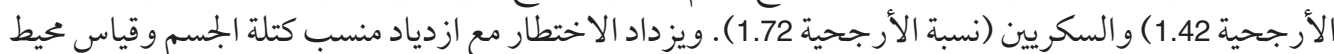

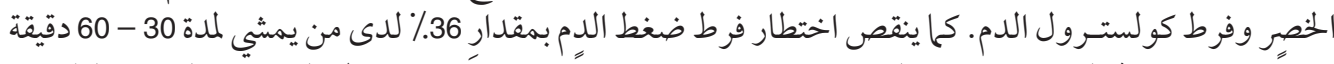

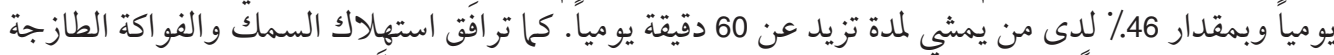

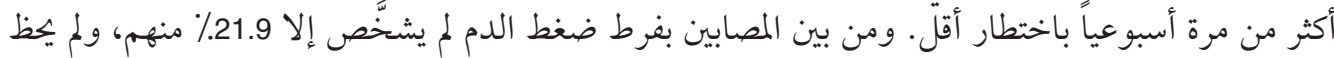
بالمعالجة الدوائية إلا من 8.8\% منهم.

ABSTRACT This study used data from the Moroccan national survey in 2000 to identify the principle risk factors for hypertension in a representative sample of the population age $20+$ years. The risk of hypertension increased steadily with age and was higher among rural residents $(O R=1.42)$ and those with diabetes $(\mathrm{OR}=1.72)$. The risk increased with increased body mass index, waist size and hypercholesterolaemia. The risk of hypertension decreased by $36 \%$ and $46 \%$ respectively for those who walked 30-60 min and $>60$ min daily. Consumption of fish and fresh fruits $1+$ times per week was associated with a lower risk. Among those with hypertension, only $21.9 \%$ were previously diagnosed and $8.8 \%$ were under medical treatment.

\section{Facteurs de risque d'hypertension dans la population adulte marocaine}

RÉSUMÉ Cette étude a exploité les données de l'enquête nationale marocaine de 2000 afin de recenser les principaux facteurs de risque d'hypertension dans un échantillon représentatif de la population âgé de 20 ans et plus. Le risque d'hypertension augmentait régulièrement avec l'âge et il était plus élevé chez les personnes vivant en milieu rural $(O R=1,42)$ et les diabétiques $(O R=1,72)$. II augmentait également si l'indice de masse corporelle, le tour de taille et l'hypercholestérolémie étaient élevés. Le risque d'hypertension diminuait de $36 \%$ chez les personnes qui marchaient entre 30 et 60 minutes par jour et de $46 \%$ chez celles qui marchaient plus de 60 minutes par jour. La consommation de poisson et de fruits frais au moins une fois par semaine était associée à un risque plus faible. Parmi les sujets hypertendus, seuls $21,9 \%$ avaient été diagnostiqués auparavant et $8,8 \%$ étaient sous traitement médical.

${ }^{1}$ Directorate of Epidemiology and Control of Diseases, Health Ministry, Rabat, Morocco (Correspondence to M.A.Tazi: matazi@hotmail.com).

${ }^{2}$ Moroccan League for the Control of Cardiovascular Diseases, Cardiology Department A, University Hospital Ibn Sina, Rabat, Morocco.

Received: 14/11/06; accepted: 28/03/07

المجلة الصحية لشرق المتوسط، منظمة الصحة العالمية، المجلد الخنامس عشر، العدد ع، 9 +. 


\section{Introduction}

It is well established that hypertension is prevalent not only among developed nations but also in developing countries [1-3]. The available data for several countries in the Eastern Mediterranean Region indicates that hypertension and its associated complications constitute an important cause of morbidity and mortality $[4,5]$. It has also been reported that well-established risk factors for cardiovascular diseases such as obesity, high serum cholesterol, diabetes and smoking pose a greater risk in those with hypertension than in those with normal blood pressure (BP) levels [6].

In Morocco, the high prevalence of hypertension is a serious public health problem. The results of the 2000 national survey on cardiovascular disease risk factors showed that $33 \%$ of individuals aged 20 years and over had hypertension [3]. With knowledge of the principle risk factors of hypertension, the segments of the population most exposed, as well as the rate of its increase in Morocco, an effective strategy to combat hypertension and its associated complications can be initiated.

The objective of this study was to identify the principal risk factors for hypertension and to determine the rate of detection, treatment and control of hypertension in a representative sample of the Moroccan population.

\section{Methods}

\section{Population and sample}

The survey conducted in 2000 used a representative sample of the Moroccan population aged $20+$ years. The sample selection was conducted through a multi-stage clustering technique. A total of 2000 people were selected from 100 communes, in clusters of 20 persons per commune. Morocco is divided into 68 administrative provinces and prefectures, each of which is composed of several communes. The number of clusters selected at random in a province or prefecture was proportional to the number of its inhabitants. Likewise, the number of clusters selected at random in urban and rural areas was proportional to the distribution of the Moroccan population in urban and rural areas, i.e. $53 \%$ and $47 \%$ respectively. Twenty (20) households were selected at random from each commune included in the survey, and 1 person aged 20+ years from each household was selected at random to take part in the survey. Pregnant women and bed-ridden terminally-ill people were excluded.

\section{Data collection}

Data collection took place at the participants' place of residence. On the first day of the survey the investigator filled in the questionnaire and took the BP and anthropometric measurements of the respondent. A blood sample was taken the next day in order to determine serum glucose, total cholesterol and high-density lipoprotein (HDL) cholesterol levels. The blood sample was centrifuged within the following hour and sent to the laboratory at the Moroccan League for the Prevention of Cardiovascular Disease at the Ibn Sina Hospital in Rabat. The methodology of this survey was reported in detail in a previous article [3].

The survey questionnaire included questions on demographic data (age, sex, place of residence, level of education), socioeconomic status (monthly income, occupation), personal and family history of hypertension, diabetes and hypercholesterolaemia, as well as questions regarding smoking habits, alcohol consumption, physical exercise and dietary habits.

Socioeconomic status was classified based on type of residence and monthly

المجلة الصحية لشرق المتوسط، منظمة الصحة العالمية، المجلد الخامس عشر، العلد ع، 9 + • 
income as: satisfactory (residence in a villa and/or monthly income $>$ US\$ 500), low (residence an apartment/traditional house and/or monthly income US\$300-500) or very low (residence in a shantytown/rural house and/or monthly income < US\$ 300).

Smoking and alcohol use were defined as: non-smoker (never smoked cigarettes), smoker (current smoker) or ex-smoker (quit smoking) and non-drinker (never drank alcohol), drinker (current drinker of alcohol) or ex-drinker (quit drinking).

Body mass index (BMI) was defined as: normal $\left(<25 \mathrm{~kg} / \mathrm{m}^{2}\right)$, overweight $(\geq 25$ to $\left.<30 \mathrm{~kg} / \mathrm{m}^{2}\right)$ or obese $\left(\geq 30 \mathrm{~kg} / \mathrm{m}^{2}\right)$. Waist size and hip size were measured at the beginning of the great trochanter and the beginning of the umbilicus respectively. Waist size was defined as: normal (men $<88 \mathrm{~cm}$; women $<80 \mathrm{~cm})$, high $($ men $\geq 88$ to $<102 \mathrm{~cm}$; women $\geq 80$ to $<88 \mathrm{~cm}$ ) or very high (men $\geq 102 \mathrm{~cm}$; women $\geq 88 \mathrm{~cm}$ ). The waist-hip ratio (WHR) as a measure of abdominal obesity was defined as: normal $($ men $<1$; women $<0.85)$ or high $($ men $\geq 1$; women $\geq 0.85$ ).

Physical activity was defined as: vigorous (accelerated breathing), moderate (rhythmic breathing) or walking. Only physical activity that lasted $>10$ minutes was considered. Each activity was measured in terms of average time spent/day (minutes).

Data about diet was based on the frequency of consumption of the main foods habitually consumed by the Moroccan population: frequently (1+ times per week), moderately ( $<1$ time per week) or rarely $(<1$ time per month).

If one of the parents of the participant was known to have hypertension, the person was considered to have a family history of hypertension. A person was considered diabetic if his/her fasting blood sugar was $\geq 1.26 \mathrm{~g} / \mathrm{L}$ and/or $\mathrm{s} /$ he took medication for diabetes. Hypercholesterolaemia was defined as cholesterol level $\geq 2.00 \mathrm{~g} / \mathrm{L}$ and low HDL cholesterol as $\leq 0.29 \mathrm{~g} / \mathrm{L}$ for men and $\leq 0.35 \mathrm{~g} / \mathrm{L}$ for women.

\section{Measurement of hypertension}

Hypertension was measured using a Vaqueztype sphygmomanometer. BP measurements were taken from both arms with the subject in a sitting position after a $10 \mathrm{~min}$ rest, after which a 3rd BP measurement was taken from the arm with the highest BP. The systolic BP and the diastolic BP values were averaged and done on the same side. A large armband was used when the width of the arm was $>33 \mathrm{~cm}$. A person was considered hypertensive with systolic $\mathrm{BP} \geq 140$ $\mathrm{mmHg}$ and/or diastolic $\mathrm{BP} \geq 90 \mathrm{mmHg}$ and/ or under treatment with antihypertensive medication. In our survey, 63 people were taking medication for hypertension.

\section{Statistical analysis}

The chi-squared test was used for the comparison of the given categories and the $t$-test for the comparison of the given means and standard deviation (SD) within groups. A non-conditional logistic regression model was utilized to analyse the association between the risk of hypertension (dependent variable) and the different risk factors of interest (independent variables). During the first analysis, a univariate analysis was conducted in order to select the covariates significantly associated with the risk of hypertension, with an alpha error less than $10 \%$. During the second analysis, a multivariate logistic regression (stepwise logistic model) was utilized in order to study the association between hypertension and the significant risk factors identified in the univariate analysis. Only those variables with a $P$-value $<0.05$ were retained in the final model.

We also examined the interactions between the different risk factors retained in

المجلة الصحية لشرق المتوسط، منظمة الصحة العالمية، المجلد الخنامس عشر، العدد ع، 9 +. 
the final model. The estimated odds ratios (OR) are presented along with the $95 \%$ confidence intervals (CI). The analysis was conducted using SPSS, version 10.

\section{Results}

In total, 1802 people (90.1\%) participated in the questionnaire survey and blood pressure/anthropometric measures ( 755 men and 1047 women); 1662 of these (92.2\%) gave blood samples the next day for serum glucose, total cholesterol and HDL cholesterol levels. The blood sample was insufficient to test HDL cholesterol in 21 people.

\section{Prevalence of hypertension}

The prevalence of hypertension was $39.6 \%$ and $33.6 \%$ after standardizing for the Moroccan population by age and by sex (men $30.2 \%$; women $37.0 \%$ ) [3]. The prevalence of hypertension increased significantly with age in both sexes (Figure 1) from 17.5\% among those aged $20-29$ years (men $17.5 \%$; women $17.4 \%$ ) up to $73.9 \%$ among those $70+$ years (men 71.4\%; women $77.2 \%$ ).

Women had significantly higher mean systolic and diastolic BP levels than men (systolic 134.0 versus $131.3 \mathrm{mmHg}, P$ $=0.017$; diastolic 78.7 versus $76.0 \mathrm{mmHg}$, $P=0.0002)$. Systolic and diastolic BP increased with age in both sexes. For men, mean BP was $123.2 / 72.2 \mathrm{mmHg}$ in those aged 20-29 years and $148.3 / 81.6 \mathrm{mmHg}$ in those $70+$ years, reflecting an increase in systolic BP of $20 \%$ and an increase in diastolic BP of $13 \%$. Among women, the mean values were $120.6 / 72.0 \mathrm{mmHg}$ and $157.0 / 87.3 \mathrm{mmHg}$ respectively in those 20-29 years and $70+$ years, reflecting a 30\% increase in systolic BP and $21 \%$ increase in diastolic BP.

The distribution of hypertensives and non-hypertensives in the population according to the measured variables is presented in Tables 1 and 2. Mean age was 50.6 years

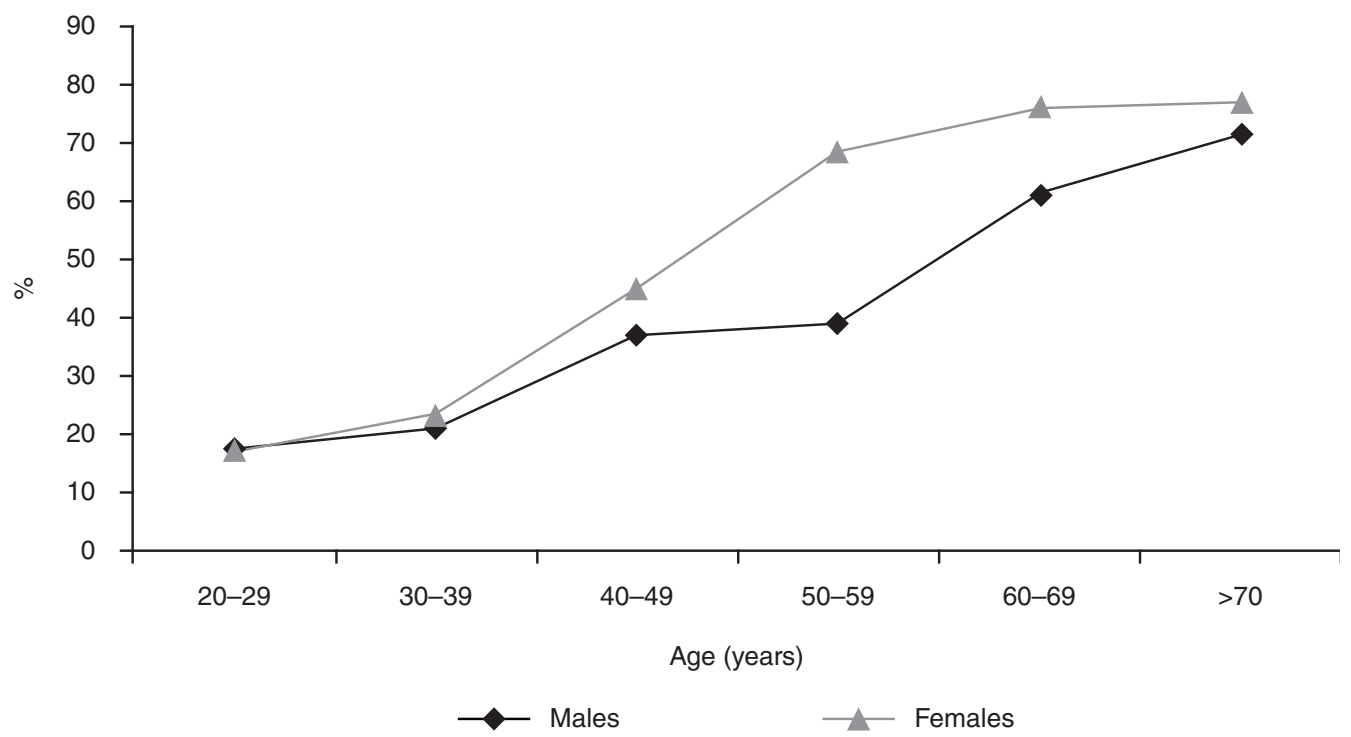

Figure 1 Prevalence of hypertension by age and sex: national health survey, Morocco 2000

المجلة الصحية لشرق المتوسط، منظمة الصحة العالمية، المجلد الخامس عشر، العلد ع، 9 +. 
Eastern Mediterranean Health Journal, Vol. 15, No. 4, 2009

Table 1 Hypertension risk factors of participants $\geq 20$ years according to hypertension status and prevalence of hypertension: national health survey, Morocco 2000

\begin{tabular}{|c|c|c|c|c|c|c|}
\hline \multirow[t]{2}{*}{ Variable } & \multicolumn{2}{|c|}{ Non-hypertensives } & \multicolumn{2}{|c|}{ Hypertensives } & \multirow{2}{*}{$\begin{array}{c}\text { Prevalence of } \\
\text { hypertension } \\
\%\end{array}$} & \multirow[t]{2}{*}{$P$-value } \\
\hline & No. & $\%$ & No. & $\%$ & & \\
\hline$\overline{\text { Age (years) }}$ & & & & & & $<0.001$ \\
\hline $20-34$ & 460 & 42.2 & 109 & 15.3 & 19.2 & \\
\hline $35-44$ & 313 & 28.7 & 134 & 18.8 & 30.0 & \\
\hline $45-54$ & 175 & 16.1 & 183 & 25.7 & 51.1 & \\
\hline $55-64$ & 81 & 7.4 & 140 & 19.6 & 63.3 & \\
\hline$\geq 65$ & 60 & 5.5 & 147 & 20.6 & 71.0 & \\
\hline Mean (SD) & \multicolumn{2}{|c|}{$38.7(13.3)$} & \multicolumn{2}{|c|}{$50.6(15.1)^{\star}$} & & \\
\hline Sex & & & & & & 0.082 \\
\hline Male & 474 & 43.5 & 281 & 39.4 & 37.2 & \\
\hline Female & 615 & 56.5 & 432 & 60.6 & 41.3 & \\
\hline Place of residence & & & & & & 0.046 \\
\hline Urban & 566 & 52.0 & 336 & 47.1 & 37.3 & \\
\hline Rural & 523 & 48.0 & 377 & 52.9 & 41.9 & \\
\hline Marital status ${ }^{a}$ & & & & & & $<0.001$ \\
\hline Single & 211 & 19.4 & 51 & 7.2 & 19.5 & \\
\hline Married & 785 & 72.1 & 528 & 74.1 & 40.2 & \\
\hline Widow & 59 & 5.4 & 117 & 16.4 & 66.5 & \\
\hline Divorced & 30 & 2.8 & 16 & 2.2 & 34.8 & \\
\hline Education level ${ }^{b}$ & & & & & & $<0.001$ \\
\hline None & 607 & 55.7 & 509 & 71.4 & 45.6 & \\
\hline Koranic school & 69 & 6.3 & 47 & 6.6 & 40.5 & \\
\hline Primary & 185 & 17.0 & 86 & 12.1 & 31.7 & \\
\hline Secondary & 175 & 16.1 & 52 & 7.3 & 22.9 & \\
\hline University & 51 & 4.7 & 18 & 2.5 & 26.1 & \\
\hline $\begin{array}{l}\text { Socioeconomic } \\
\text { status }\end{array}$ & & & & & & 0.677 \\
\hline Satisfactory & 26 & 2.4 & 17 & 2.4 & 39.5 & \\
\hline Low & 473 & 43.4 & 296 & 41.5 & 38.5 & \\
\hline Very low & 590 & 54.2 & 400 & 56.1 & 40.4 & \\
\hline $\begin{array}{l}\text { Family history of } \\
\text { hypertension }\end{array}$ & & & & & & 0.988 \\
\hline No & 961 & 88.2 & 630 & 88.4 & 39.6 & \\
\hline Yes & 128 & 11.8 & 83 & 11.6 & 39.3 & \\
\hline Cigarette smoking & & & & & & $<0.001$ \\
\hline Never smoked & 826 & 75.8 & 576 & 80.8 & 41.1 & \\
\hline Current smoker & 179 & 16.4 & 65 & 9.1 & 26.6 & \\
\hline Ex-smoker & 84 & 7.7 & 72 & 10.1 & 46.2 & \\
\hline
\end{tabular}


Table 1 Hypertension risk factors of participants aged 20 years and over according to hypertension status and prevalence of hypertension: national health survey, Morocco 2000 (continued)

\begin{tabular}{|c|c|c|c|c|c|c|}
\hline \multirow[t]{2}{*}{ Variable } & \multicolumn{2}{|c|}{ Non-hypertensives } & \multicolumn{2}{|c|}{ Hypertensives } & \multirow{2}{*}{$\begin{array}{c}\text { Prevalence of } \\
\text { hypertension } \\
\%\end{array}$} & \multirow[t]{2}{*}{$P$-value } \\
\hline & No. & $\%$ & No. & $\%$ & & \\
\hline Alcohol drinking & & & & & & 0.066 \\
\hline Never drank & 958 & 88.0 & 650 & 91.2 & 40.4 & \\
\hline Current drinker & 36 & 3.3 & 14 & 2.0 & 28.0 & \\
\hline Ex-drinker & 95 & 8.7 & 49 & 6.9 & 34.0 & \\
\hline \multicolumn{6}{|l|}{$\begin{array}{l}\text { Moderate physical } \\
\text { activity (min/day) }\end{array}$} & 0.004 \\
\hline$\leq 120$ & 477 & 43.8 & 365 & 51.2 & 43.3 & \\
\hline $121-240$ & 300 & 27.5 & 184 & 25.8 & 38.0 & \\
\hline$>240$ & 312 & 28.7 & 164 & 23.0 & 34.5 & \\
\hline Mean (SD) & \multicolumn{2}{|c|}{$187.6(161.5)^{\star}$} & \multicolumn{2}{|c|}{$159.9(157.0)$} & & \\
\hline \multicolumn{6}{|l|}{$\begin{array}{l}\text { Intense physical } \\
\text { activity (min/day) }\end{array}$} & $<0.001$ \\
\hline$\leq 60$ & 860 & 79.0 & 607 & 85.1 & 41.4 & \\
\hline$>60$ & 229 & 21.0 & 106 & 14.9 & 31.6 & \\
\hline Mean (SD) & \multicolumn{2}{|c|}{$62.5(135.9)^{*}$} & \multicolumn{2}{|c|}{$39.2(105.3)$} & & \\
\hline \multicolumn{5}{|l|}{ Walking (min/day) } & & $<0.001$ \\
\hline$\leq 30$ & 313 & 28.8 & 298 & 41.8 & 48.8 & \\
\hline $31-60$ & 301 & 27.6 & 175 & 24.5 & 36.8 & \\
\hline $61-120$ & 261 & 24.0 & 137 & 19.2 & 34.4 & \\
\hline$>120$ & 214 & 19.7 & 103 & 14.4 & 32.5 & \\
\hline Mean (SD) & \multicolumn{2}{|c|}{$88.5(97.5)^{\star}$} & \multicolumn{2}{|c|}{$69.1(86.8)$} & & \\
\hline \multicolumn{3}{|l|}{$B M I^{a}\left(\mathrm{~kg} / \mathrm{m}^{2}\right)$} & & & & $<0.001$ \\
\hline$<25$ & 697 & 64.0 & 308 & 43.2 & 30.6 & \\
\hline 25-29 & 269 & 24.7 & 234 & 32.8 & 46.5 & \\
\hline$\geq 30$ & 121 & 11.1 & 168 & 23.6 & 58.1 & \\
\hline Mean (SD) & \multicolumn{2}{|c|}{$24.2(4.4)$} & \multicolumn{2}{|c|}{$26.7(5.3)^{\star}$} & & \\
\hline \multicolumn{3}{|l|}{ Waist size $(\mathrm{cm})$} & & & & $<0.001$ \\
\hline Normal & 737 & 67.7 & 296 & 41.5 & 28.7 & \\
\hline High & 171 & 15.7 & 151 & 21.2 & 46.9 & \\
\hline Very high & 177 & 16.3 & 266 & 37.3 & 60.0 & \\
\hline Mean (SD) & \multicolumn{2}{|c|}{$80.8(11.1)$} & \multicolumn{2}{|c|}{$88.6(12.2)^{\star}$} & & \\
\hline \multicolumn{3}{|l|}{ Waist-hip ratioc } & & & & $<0.001$ \\
\hline Normal & 880 & 81.1 & 468 & 65.6 & 34.7 & \\
\hline High & 205 & 18.9 & 245 & 34.4 & 54.4 & \\
\hline Mean (SD) & 0.8 & $0.08)$ & $0.8 \varepsilon$ & $.08)^{*}$ & & \\
\hline
\end{tabular}


Eastern Mediterranean Health Journal, Vol. 15, No. 4, 2009

\begin{tabular}{|c|c|c|c|c|c|c|}
\hline \multirow[t]{2}{*}{ Variable } & \multicolumn{2}{|c|}{ Non-hypertensives } & \multicolumn{2}{|c|}{ Hypertensives } & \multirow{2}{*}{$\begin{array}{c}\text { Prevalence of } \\
\text { hypertension } \\
\%\end{array}$} & \multirow[t]{2}{*}{$P$-value } \\
\hline & No. & $\%$ & No. & $\%$ & & \\
\hline $\begin{array}{l}\text { Fasting blood } \\
\operatorname{sugar}^{d}(g / L)\end{array}$ & & & & & & $<0.001$ \\
\hline$<1.26$ & 957 & 87.9 & 565 & 79.2 & 37.1 & \\
\hline$\geq 1.26$ & 48 & 4.4 & 91 & 12.8 & 65.5 & \\
\hline Missing data & 84 & 7.7 & 57 & 8.0 & 40.4 & \\
\hline Mean (SD) & \multicolumn{2}{|c|}{$0.94(0.34)$} & \multicolumn{2}{|c|}{$1.06(0.48)^{*}$} & & \\
\hline Cholesterold $^{(g / L)}$ & & & & & & $<0.001$ \\
\hline$<2.0$ & 750 & 68.9 & 349 & 48.9 & 31.8 & \\
\hline $2.0-2.2$ & 122 & 11.2 & 113 & 15.8 & 48.1 & \\
\hline$>2.2$ & 133 & 12.2 & 195 & 27.3 & 59.5 & \\
\hline Mean (SD) & \multicolumn{2}{|c|}{$1.76(0.39)$} & \multicolumn{2}{|c|}{$2.00(0.46)^{*}$} & & \\
\hline Missing data & 84 & 7.7 & 56 & 7.9 & 40.0 & \\
\hline \multicolumn{6}{|l|}{$\begin{array}{l}\text { HDL cholesterole } \\
(\mathrm{g} / \mathrm{L})\end{array}$} & 0.462 \\
\hline Normal & 955 & 87.7 & 610 & 85.6 & 39.0 & \\
\hline Low & 42 & 3.9 & 34 & 4.8 & 44.7 & \\
\hline Mean (SD) & \multicolumn{2}{|c|}{$0.50(0.15)$} & \multicolumn{2}{|c|}{$0.51(0.14)$} & & \\
\hline Missing data & 92 & 8.4 & 69 & 9.7 & 42.9 & \\
\hline
\end{tabular}

\section{${ }^{*} P<0.001$.}

aUnknown for 5 people; bunknown for 3 people; cunknown for 4 people.

${ }^{d} 1662$ people consented to give a blood sample.

${ }^{e}$ Blood sample was insufficient to test high-density lipoprotein cholesterol in 21 people.

Mean (SD) systolic/diastolic blood pressure was $118.5(10.8) / 69.4(8.7) \mathrm{mmHg}$ in the nonhypertensive group and $154.9(22.0) / 90.1$ (14.1) $\mathrm{mmHg}$ in the hypertensive group $(\mathrm{P}<0.01)$.

$S D=$ standard deviation; $H D L=$ high-density lipoprotein .

and 38.7 years respectively among those with and without hypertension $(P<0.001)$. The mean systolic and diastolic BP levels were 154.9 (SD 43.1)/90.1 (SD 27 6) $\mathrm{mmHg}$ and 118.5 (SD 21.2)/69.4 (SD 17.4) $\mathrm{mmHg}$ respectively among those with and without hypertension.

\section{Risk factors for hypertension}

The univariate analysis indicated an increased risk of hypertension among rural residents, widowers and individuals with low levels of education. The risk of hypertension was positively associated with age, BMI, waist size and WHR. Hypertension was also positively associated with diabetes, high cholesterol and lowered levels of HDL cholesterol. Those without hypertension more frequently consumed fish, lamb, chicken, eggs, beans and dried fruits. The risk of hypertension was lower among those who smoked cigarettes, drank alcohol and participated in more physical activity or walking, and was not based on socioeconomic status or family history of hypertension.

The multivariate analysis indicated that age, place of residence, BMI, waist size, diabetes, high cholesterol, walking, the consumption of fish and fresh fruits were all independent risk factors associated with

المجلة الصحية لشرق المتوسط، منظمة الصحة العالمية، المجلد الخنامس عشر، العدد ع، 9 . • ب 


\begin{tabular}{|c|c|c|c|c|c|c|}
\hline \multirow{2}{*}{$\begin{array}{l}\text { Frequency } \\
\text { of food } \\
\text { consumption }\end{array}$} & \multicolumn{2}{|c|}{ Non-hypertensives } & \multicolumn{2}{|c|}{ Hypertensives } & \multirow{2}{*}{$\begin{array}{c}\text { Prevalence of } \\
\text { hypertension } \\
\%\end{array}$} & \multirow[t]{2}{*}{$P$-value } \\
\hline & No. & $\%$ & No. & $\%$ & & \\
\hline Beef & & & & & & 0.644 \\
\hline Rarely & 286 & 26.3 & 203 & 28.5 & 41.5 & \\
\hline Moderately & 181 & 16.6 & 117 & 16.4 & 39.3 & \\
\hline Frequently & 622 & 57.1 & 393 & 55.1 & 38.7 & \\
\hline Lamb & & & & & & 0.002 \\
\hline Rarely & 474 & 43.5 & 370 & 51.9 & 43.8 & \\
\hline Moderately & 231 & 21.2 & 134 & 18.8 & 36.7 & \\
\hline Frequently & 384 & 35.3 & 209 & 29.3 & 35.2 & \\
\hline Chicken & & & & & & 0.004 \\
\hline Rarely & 197 & 18.1 & 159 & 22.3 & 44.7 & \\
\hline Moderately & 246 & 22.6 & 187 & 26.2 & 43.2 & \\
\hline Frequently & 646 & 59.3 & 367 & 51.5 & 36.2 & \\
\hline Fish & & & & & & $<0.001$ \\
\hline Rarely & 237 & 21.8 & 232 & 32.5 & 49.5 & \\
\hline Moderately & 228 & 20.9 & 160 & 22.4 & 41.2 & \\
\hline Frequently & 624 & 57.3 & 321 & 45.0 & 34.0 & \\
\hline Eggs & & & & & & $<0.001$ \\
\hline Rarely & 188 & 17.3 & 178 & 25.0 & 48.6 & \\
\hline Moderately & 166 & 15.2 & 100 & 14.0 & 37.6 & \\
\hline Frequently & 735 & 67.5 & 435 & 61.0 & 37.2 & \\
\hline Fresh vegetables & & & & & & 0.348 \\
\hline Rarely & 10 & 0.9 & 12 & 1.7 & 54.5 & \\
\hline Moderately & 19 & 1.7 & 11 & 1.5 & 36.7 & \\
\hline Frequently & 1060 & 97.3 & 690 & 96.8 & 39.4 & \\
\hline Beans & & & & & & $<0.001$ \\
\hline Rarely & 351 & 32.2 & 295 & 41.4 & 45.7 & \\
\hline Moderately & 307 & 28.2 & 164 & 23.0 & 34.8 & \\
\hline Frequently & 431 & 39.6 & 254 & 35.6 & 37.1 & \\
\hline Fresh fruits & & & & & & 0,015 \\
\hline Rarely & 91 & 8.4 & 81 & 11.4 & 47.1 & \\
\hline Moderately & 132 & 12.1 & 108 & 15.1 & 45.0 & \\
\hline Frequently & 866 & 79.5 & 524 & 73.5 & 37.7 & \\
\hline Dried fruits & & & & & & 0.014 \\
\hline Rarely & 719 & 66.0 & 515 & 72.2 & 41.7 & \\
\hline Moderately & 196 & 18.0 & 112 & 15.7 & 36.4 & \\
\hline Frequently & 174 & 16.0 & 86 & 12.1 & 33.1 & \\
\hline
\end{tabular}


Eastern Mediterranean Health Journal, Vol. 15, No. 4, 2009

\begin{tabular}{|c|c|c|c|c|c|c|}
\hline \multirow{2}{*}{$\begin{array}{l}\text { Frequency } \\
\text { of food } \\
\text { consumption }\end{array}$} & \multicolumn{2}{|c|}{ Non-hypertensives } & \multicolumn{2}{|c|}{ Hypertensives } & \multirow{2}{*}{$\begin{array}{c}\text { Prevalence of } \\
\text { hypertension } \\
\%\end{array}$} & \multirow[t]{2}{*}{$P$-value } \\
\hline & No. & $\%$ & No. & $\%$ & & \\
\hline Olive oil & & & & & & 0.982 \\
\hline Rarely & 279 & 25.6 & 184 & 25.8 & 39.7 & \\
\hline Moderately & 83 & 7.6 & 53 & 7.4 & 39.0 & \\
\hline Frequently & 727 & 66.8 & 476 & 66.8 & 39.6 & \\
\hline Vegetable oil & & & & & & 0.338 \\
\hline Rarely & 54 & 5.0 & 40 & 5.6 & 42.6 & \\
\hline Moderately & 14 & 1.3 & 14 & 2.0 & 50.0 & \\
\hline Frequently & 1021 & 93.8 & 659 & 92.4 & 39.2 & \\
\hline Butter & & & & & & 0.526 \\
\hline Rarely & 438 & 40.2 & 306 & 42.9 & 41.1 & \\
\hline Moderately & 145 & 13.3 & 95 & 13.3 & 39.6 & \\
\hline Frequently & 506 & 46.5 & 312 & 43.8 & 38.1 & \\
\hline Dairy & & & & & & 0.674 \\
\hline Rarely & 223 & 20.5 & 155 & 21.7 & 41.0 & \\
\hline Moderately & 144 & 13.2 & 102 & 14.3 & 41.5 & \\
\hline Frequently & 722 & 66.3 & 456 & 64.0 & 38.7 & \\
\hline Tea & & & & & & 0.757 \\
\hline Rarely & 40 & 3.7 & 31 & 4.3 & 43.7 & \\
\hline Moderately & 12 & 1.1 & 9 & 1.3 & 42.9 & \\
\hline Frequently & 1037 & 95.2 & 673 & 94.4 & 39.4 & \\
\hline Coffee & & & & & & 0.877 \\
\hline Rarely & 642 & 59.0 & 423 & 59.3 & 39.7 & \\
\hline Moderately & 98 & 9.0 & 67 & 9.4 & 40.6 & \\
\hline Frequently & 349 & 32.0 & 223 & 31.3 & 39.0 & \\
\hline
\end{tabular}

hypertension (Table 3). The risk of hypertension increased with age. Hypertension was 8 times more likely among those age 65+ years compared with those $20-34$ years old $(P<0.001)$. The risk of hypertension increased among rural residents $(\mathrm{OR}=1.42)$. The risk of hypertension increased by $36 \%$ among overweight individuals and 79\% among obese individuals, compared with those of a normal weight. Similarly, the risk of hypertension increased by $77 \%$ and $130 \%$ respectively when waist size was high and very high. The risk of hypertension was 1.7 times higher among diabetics and
1.4 times and 1.9 times higher respectively when cholesterol levels were $2.0-2.2 \mathrm{~g} / \mathrm{L}$ or $>2.2 \mathrm{~g} / \mathrm{L}$.

Individuals who consumed fish and fresh fruits at least once per week were at low risk for hypertension ( $43 \%$ and $31 \%$, respectively) than those who consumed these items less than once per month (the reference category).

Walking was also associated with a low risk of hypertension. The decrease in risk was $36 \%$ for those who walked $30-60 \mathrm{~min} /$ day and $46 \%$ for those who walked more than 60 min daily. 


\begin{tabular}{|c|c|c|c|}
\hline \multirow[t]{2}{*}{ Variable } & \multicolumn{2}{|c|}{ Multivariate analysis } & \multirow{2}{*}{$\begin{array}{l}\text { Odds ratio } \\
(95 \% \mathrm{Cl})\end{array}$} \\
\hline & $P$-value step 0 & $P$-value final & \\
\hline Age (years) & $<0.001$ & $<0.001$ & \\
\hline $20-34$ & & & 1 \\
\hline $35-44$ & & & $1.41(1.03-1.93)$ \\
\hline $45-54$ & & & $3.21(2.33-4.43)$ \\
\hline $55-64$ & & & $5.53(3.79-8.06)$ \\
\hline$\geq 65$ & & & $8.00(5.37-11.9)$ \\
\hline Place of residence & 0.017 & 0.005 & \\
\hline Urban & & & 1 \\
\hline Rural & & & $1.42(1.11-1.81)$ \\
\hline Sex & 0.210 & 0.526 & \\
\hline Marital status & 0.355 & 0.417 & \\
\hline Education level & 0.849 & 0.912 & \\
\hline Cigarette smoking & 0.337 & 0.473 & \\
\hline Alcohol drinking & 0.745 & 0.763 & \\
\hline Walking (min/day) & $<0.001$ & 0.001 & \\
\hline$\leq 30$ & & & 1 \\
\hline $31-60$ & & & $0.64(0.48-0.85)$ \\
\hline $61-120$ & & & $0.53(0.39-0.71)$ \\
\hline$>120$ & & & $0.54(0.39-0.75)$ \\
\hline $\begin{array}{l}\text { Moderate physical } \\
\text { activity } \\
\text { Intense physical }\end{array}$ & 0.913 & 0.600 & \\
\hline activity & 0.991 & 1.000 & \\
\hline$B M I\left(\mathrm{~kg} / \mathrm{m}^{2}\right)$ & 0.021 & 0.023 & \\
\hline$<25$ & & & 1 \\
\hline $25-29$ & & & $1.36(1.00-1.83)$ \\
\hline$\geq 30$ & & & $1.79(1.17-2.74)$ \\
\hline Waist size $(\mathrm{cm})$ & 0.001 & $<0.001$ & \\
\hline Normal & & & 1 \\
\hline High & & & $1.77(1.28-2.44)$ \\
\hline Very high & & & $2.30(1.59-3.31)$ \\
\hline Waist-hip-ratio & 0.991 & 0.955 & \\
\hline Diabetes & 0.024 & 0.011 & \\
\hline No & & & 1 \\
\hline Yes & & & $1.72(1.12-2.63)$ \\
\hline Cholesterol $(\mathrm{g} / \mathrm{L})$ & 0.001 & 0.0003 & \\
\hline$<2.0$ & & & 1 \\
\hline 2.0-2.19 & & & $1.39(1.00-1.93)$ \\
\hline _ $\geqq 2.20$ & ---- & $-\ldots$ & $1.88(1.39-2.55)$ \\
\hline
\end{tabular}




\begin{tabular}{|c|c|c|c|}
\hline $\begin{array}{l}\text { Table } 3 \text { Associa } \\
\text { factors: nationa }\end{array}$ & $\begin{array}{l}\text { etween hyperte } \\
\text { th survey, Moro }\end{array}$ & $\begin{array}{l}\text { ision and po } \\
\text { co } 2000 \text { (cor }\end{array}$ & $\begin{array}{l}\text { ntial risk } \\
\text { luded) }\end{array}$ \\
\hline Variable & Mulitvaria & analysis & Odds ratio \\
\hline & $P$-value step 0 & $P$-value final & (95\% Cl) \\
\hline Consumption of: & & & \\
\hline Fish & 0.005 & $<0.001$ & 1 \\
\hline Rarely & & & $0.85(0.62-1.17)$ \\
\hline Moderately & & & $0.57(0.43-0.76$ \\
\hline Frequently & & & \\
\hline Fresh fruits & 0.065 & 0.015 & \\
\hline Rarely & & & 1 \\
\hline Moderately & & & $1.07(0.67-1.68)$ \\
\hline Frequently & & & $0.69(0.48-1.01)$ \\
\hline Lamb & 0.190 & 0.129 & \\
\hline Chicken & 0.200 & 0.130 & \\
\hline Eggs & 0.225 & 0.176 & \\
\hline Beans & 0.272 & 0.186 & \\
\hline Dried fruits & 0.990 & 0.856 & \\
\hline
\end{tabular}

Sex, level of education, marital status, drinking alcohol, smoking and moderate or intense physical activity were not significantly associated with the risk of hypertension.

\section{Treatment of hypertension}

In our survey, $156(21.9 \%)$ of those with hypertension had been diagnosed beforehand (men 13.5\%; women 27.3\%) and 63 of those (40.4\%) were taking antihypertension medication (men 26.3\%; women 44.9\%). Only $8 / 63(12.7 \%)$ cases with hypertension and under antihypertension treatment were controlled (i.e. $\mathrm{BP}<140 / 90 \mathrm{mmHg}$ ) (men $20.0 \%$; women $11.3 \%$ ). Thus of those with hypertension, only $8.8 \%$ were under medical treatment (men 3.6\%; women $12.3 \%$ ) and only $1.1 \%$ were controlled (men $0.7 \%$; women $1.4 \%$ ).

\section{Discussion}

Our study has the advantage of using a representative sample of the Moroccan popula- tion and including a number of potential important risk factors of hypertension.

Similar to other studies, the risk of hypertension increased with age [7-12]. This progressive increase is linked with rising systolic BP, increasing with age to an average value close to $140 \mathrm{mmHg}$ in the 7th decade [13].

The rural population was more at risk of hypertension. In the first report we suggested a possible link between stress and rural residence, the majority of such residents work in agriculture, due to the drought in Morocco over the past 2 decades [3]. Stress is known to be a risk factor of hypertension, but other studies are necessary to confirm or refute this [14].

The prevalence of hypertension in our survey was slightly higher among women [3]. However, multivariate analysis indicated that women did not have a higher risk of hypertension than men. Similarly, marital status, level of education and socioeconomic

المجلة الصحية لشرق المتوسط، منظمة الصحة العالمية، المجلد الخنامس عشر، العدد ع، 9 +. 
status, as defined in our study, were not significant risk factors for hypertension in the multivariate analysis. But in other studies a high risk of hypertension has been associated with low education level [10] and with a higher socioeconomic status [15].

Many studies have examined the effect of smoking on the prevalence of hypertension. In our study, smoking was not a significant risk factor for hypertension. A similar result was found in the study of Mohsen Ibrahim in Egypt [16]. Certain studies indicate that smokers and ex-smokers are more at risk [17-19]. It has also been suggested that BP among ex-smokers is higher than that of smokers as well as those who have never smoked [20]. The increased risk of hypertension among ex-smokers has been attributed to the number of cigarettes smoked daily prior to cessation of smoking and not to the duration of smoking [21]. Recently, it was suggested that smoking was associated with an increase in systolic BP among older men and not among women [22] and an increase in the incidence of hypertension [23]. Other studies have indicated an inverse relationship between smoking and $\mathrm{BP}$ and the risk of hypertension $[7,14,20]$. These contradictory results indicate that the relationship between smoking and hypertension is complicated. This is further complicated by the finding that those who smoke may consume a different diet from those who do not smoke [24]. However, it is important to note that the relationship between other factors may contribute to the risk of hypertension, such as the age at which one starts smoking and the time elapsed since smoking cessation.

The positive association between obesity and hypertension observed in our study has been reported in numerous studies $[8,10-12,14,16,19,25,26]$. The Framingham study indicated that $70 \%$ of new-onset hypertension may be attributed to obesity or weight gain [25]. The mechanism through which obesity and BP are related has yet to be fully elucidated. Resistance to insulin and peripheral hyperinsulinaemia due to overweight and obesity play a role in the development of hypertension [27].

Waist size reflects abdominal obesity and is considered a good indicator of obesity. The positive association observed in our study between waist size and hypertension has been reported in other studies $[12,15,16,28]$. Some of those studies indicated that abdominal obesity was a risk factor for hypertension independently of BMI $[12,15,28]$. Ledoux reported that obesity (BMI) and the abdominal distribution of fat (waist size or WHR) are equally associated with the presence of hypertension [29]. Similar to the study by Perez [14], physical activity was not associated with the risk of hypertension in our study. However, walking did seem to have a protective role against hypertension. In the study of Singh et al. in India, lower physical activity was weakly but significantly associated with hypertension [15].

The results of our study confirm those of similar studies which indicate that there is an increased risk of hypertension among diabetics $[14,16,26]$ and those with hypercholesterolaemia [16]. The association between hypercholesterolaemia and the risk of hypertension may explain some of the mechanisms: first, hypertension and hypercholesterolaemia are linked to atherosclerosis which is associated with reduced vascular compliance [30]; secondly, high cholesterol is associated with vascular diseases, which are linked to changes in the endothelium [31,32]; thirdly, food rich in fat and cholesterol may cause a disturbance in the metabolism of prostaglandins [33]; and fourthly, hypertension and hypercholesterolemia are present in familial dyslipidaemia and hypertension [34]. 
In our study, drinking alcohol was not significantly associated with hypertension, similar to the results of Perez et al. [14], although other studies have found an association $[7,25,35]$. A Japanese study found not only a dose-effect relationship between the quantity of alcohol consumed and the level of BP but also a higher prevalence of hypertension among heavy drinkers of alcohol [35]. Another study showed that BMI and the consumption of alcohol are strong predictors of hypertension [25].

The frequent consumption of fresh fruit and fish seems to have a protective role against hypertension. Sacks et al. suggested that consumption of fresh fruits and vegetables are associated with a low risk of hypertension [36]. A number of studies indicate that fish has a protective effect against coronary artery disease [37-39] but there are few available data concerning the relationship between the consumption of fish and BP. Similar to the study conducted by Perez et al [14], the consumption of animal products high in fat were not found to be associated with an increased risk of hypertension. Concerning the relationship between the risk of hypertension and the other foods, few data are available in the international literature.

In this study, family history of hypertension was not found to be associated with hypertension. This contrasts with a number of other studies indicating a positive association suggesting a hereditary component to hypertension $[14,19,26]$.

The detection rate for hypertension ( 1 in 5 patients) and the proportion under treatment for hypertension (26\% for men and $45 \%$ for women) remains very low in Morocco. This situation is of further concern because only 1 in 8 patients under medical treatment had a normal level of BP. According to our study, of 100 people with hypertension only 1 had their BP controlled. The reduction in the prevalence of hypertension and its associated complications requires the reduction of the known risk factors and early and adequate assistance for hypertensive patients. It is important to educate the population, in particular those at high risk (obese, diabetic, sedentary lifestyle, etc), who must be identified and assisted.

Our study had certain limitations. The measures of BP were only taken during 1 visit, thus it was not possible to confirm with complete certainty the presence of hypertension among those with elevated BP [18]. The number of cigarettes smoked per day, the quantity of alcohol consumed and the duration of smoking and drinking alcohol were not measured. The food consumption evaluation, which measured the frequency of consumption of certain foods, was an imprecise measure and may have introduced bias, as people in the survey may not have remembered or may not have wanted to declare what they ate and because the quantity of the different foods consumed and nutrient content (carbohydrates, fats, etc.) were not taken into account.

\section{Conclusions}

The study results suggest that to reduce the prevalence of hypertension among the Moroccan population necessitates a reduction of certain risk factors, notably obesity and high serum cholesterol, but also the promotion of a healthy lifestyle with regular walking and a balanced diet rich in fresh fruits and fish.

The percentage of individuals with hypertension who are diagnosed and treated is low in Morocco and greater efforts are needed to improve the situation in order to reduce the complications associated with hypertension, most notably stroke. 


\section{References}

1. Gharbi M et al. Niveau des facteurs de risque cardiovasculaire dans la population urbaine et rurale du Cap-Bon: Tunisie [Cardiovascular risk factors in the urban and rural populations of the Cap-Bon : Tunisie]. Revue d'épidémiologie et de santé publique, 1996, 44:125-32.

2. Berrios $X$ et al. Distribution and prevalence of major risk factors of noncommunicable diseases in selected countries: the WHO Inter-Health Programme. Bulletin of the World Health Organization, 1997, 75:99-108.

3. Tazi MA et al. Prevalence of the main cardiovascular risk factors in Morocco: results of a national survey, 2000. Journal of hypertension, 2003, 21:897-903.

4. Alwan A. Prevention and management of hypertension. Alexandria, WHO Regional Office for the Eastern Mediterranean, 1996 (EMRO Technical Publication Series, No. 23).

5. Alwan A. Prevention and control of cardiovascular diseases. Alexandria, WHO Regional Office for the Eastern Mediterranean, 1995 (EMRO Technical Publication Series, No. 22).

6. Laurenzi M et al. On behalf of the Gubbio study group. Multiple risk factors in hypertension: results from the Gubbio study. Journal of hypertension, 1990, 8:7-12.

7. Hu FB et al. Body mass index and cardiovascular risk factors in a rural Chinese. American journal of epidemiology, 2000, 151:88-97.

8. Kawada T. Body mass index is a good predictor of hypertension and hyperlipidemia in a rural Japanese population. International journal of obesity, 2002, 26:725-9.

9. Brown CD et al. Body mass index and the prevalence of hypertension and dyslepidemia. Obesity research, 2000, 8:605-19.
10. Gus I et al. Prevalence, awareness, and control of systemic arterial hypertension in the state of Rio Grande do Sul. Arquivos brasileiros de cardiologia, 2004 83:429-33.

11. Shah SM et al. Hypertension and its determinants among adults in high mountain villages of the Northern Areas of Pakistan. Journal of human hypertension, 2001, 15:107-12.

12. Jo I et al. Prevalence, awareness, treatment, control and risk factors of hypertension in Korea: the Ansan study. Journal of hypertension, 2001, 19:1523-32.

13. Whelton PK. Epidemiology of hypertension. Lancet, 1994, 344:101-6.

14. Perez LH et al. Relation between overweight, diabetes, stress and hypertension: A case-control study in Yarumal-Antioquia, Colombia. European journal of epidemiology, 2001, 17:275-80.

15. Singh RB et al. Epidemiological study of hypertension and its determinants in an urban population of North India. Journal of human hypertension, 1997, 11:679-85.

16. Ibrahim MM et al. Cardiovascular risk factors in normotensive and hypertensive Egyptians. Journal of hypertension, 2001, 19:1933-40.

17. Aday LA et al. Estimating the risk and prevalence of hypertension in a suburban area of Beijing. Journal of community health, 1994, 19:331-41.

18. Halimi JM et al. The risk of hypertension in men: direct and indirect effects of chronic smoking. Journal of hypertension, 2002, 20:187-93.

19. Shey Wiysonge CU, Ngu Blackett K, Mbuagbaw JN. Risk factors and complications of hypertension in Yaounde, Cameroon. Cardiovascular journal of South Africa, 2004, 15:215-9. 
20. Seltzer CC. Effect of smoking on blood pressure. American heart journal, 1974, 87:558-64.

21. Gueyffier FJ et al. Identification of risk factors in hypertensive patients. Contribution of randomized controlled trials through an individual patient database. Circulation, 1999, 100:e88-94.

22. Primatesta $P$ et al. Association between smoking and blood pressure: evidence from the health survey for England. $\mathrm{Hy}$ pertension, 2001, 37:187-93.

23. Lee DH et al. Effects of smoking cessation on changes in blood pressure and incidence of hypertension: a 4-year followup-study. Hypertension, 2001, 37:194-8.

24. Margetts BM, Jackson AA. Interaction between people's diet and their smoking habits. British medical journal, 1993, 307:1381-4.

25. Kannel WB et al. The relation of adiposity to blood pressure and development of hypertension: the Framingham Study. Annals of internal medicine, 1967, 67:48-59.

26. Tozawa $M$ et al. Multiple risk factor clustering of hypertension in a screened cohort. Journal of hypertension, 2000, 18:1379-85.

27. Plaisted CS, Istfan NW. Metabolic abnormalities of obesity. In: Blackburn GL, Kanders BS, eds. Obesity pathophysio/ogy, psychology, and treatment. New York, Chapman and Hall, 1994:80-97.

28. Thomas GN et al. Impact of sex-specific body composition on cardiovascular risk factors: the Hong Kong Cardiovascular Risk Factor Study. Metabolism, 2006, 55:563-9.

29. Ledoux $M$ et al. Correlation between cardiovascular disease risk factors and simple anthropometric measures. Canadian Heart Survey Research Group. Canadian Medical Association journal, 1997, 157(Suppl. 1):S46-53.
30. Bulpitt CJ, Hodes C, Everitt MG. The relationship between blood pressure and biochemical risk factors in a general population. British journal of preventive and social medicine, 1976, 30:158-62.

31. Henry PD. Hyperlipidimic arterial dysfunction. Circulation, 1990, 7:697-9.

32. Vita JA et al. Coronary vasomotor response to acetylcholine relates to risk factors for coronary artery disease. Circulation, 1990, 81:491-7.

33. Galli C. Dietary influence on prostaglandin synthesis. Advances in nutritional research, 1980, 3:95-126.

34. Williams RR et al. Familial dyslipidemic hypertension. Evidence from 58 Utah families for a syndrome present in approximately $12 \%$ of patients with essential hypertension. Journal of the American Medical Association, 1988, 259:3579-86.

35. Tsuruta M et al. Association between alcohol intake and development of hypertension in Japanese normotensive men: 12-year follow-up study. American journal of hypertension, 2000, 13:482-7.

36. Sacks FM et al. Effects on blood pressure of reduced dietary sodium and the dietary approaches to stop hypertension (DASH) diet. New England journal of medicine, 2001, 344:3-10.

37. Kromann N, Green A. Epidemiological studies in the Upernavik district, Greenland. Acta medica scandinavica, 1980, 208:401-6.

38. Kromhout D, Bosschierter EB, Coulander $\mathrm{CL}$. The inverse relationship between fish consumption and 20-year mortality rate from coronary heart disease. New England journal of medicine, 1985 , 312:1205-9.

39. Norell SE et al. Fish consumption and mortality from coronary heart disease. British medical journal, 1986, 293:426. 